Témoigner Témoigner. Entre histoire et mémoire

Getuigen Revue pluridisciplinaire de la Fondation Auschwitz

$126 \mid 2018$

Questions sur l'avenir du travail de mémoire

\title{
Pédagogie des lieux de mémoire et responsabilisation
}

'Wat heeft dat met mij te maken?' Memoriaalpedagogie en empowerment

in Mauthausen

\section{Yariv Lapid}

Traducteur : Emilie Syssau

\section{(2) OpenEdition \\ 1 Journals}

Édition électronique

URL : https://journals.openedition.org/temoigner/7238

DOI : $10.4000 /$ temoigner.7238

ISSN : 2506-6390

Traduction(s) :

'Wat heeft dat met mij te maken?' - URL : https://journals.openedition.org/temoigner/7280 [nl]

Éditeur :

Éditions du Centre d'études et de documentation Mémoire d'Auschwitz, Éditions Kimé

Édition imprimée

Date de publication : 2 avril 2018

Pagination : 79-91

ISBN : 978-2-930953-06-9

ISSN : 2031-4183

Référence électronique

Yariv Lapid, « Pédagogie des lieux de mémoire et responsabilisation», Témoigner. Entre histoire et mémoire [En ligne], 126 | 2018, mis en ligne le 20 janvier 2022, consulté le 04 février 2022. URL : http:// journals.openedition.org/temoigner/7238; DOI : https://doi.org/10.4000/temoigner.7238 


\section{Pédagogie des lieux de mémoire et responsabilisation}

$\rightarrow$ Yariv Lapid
Après des étapes à Yad
Vashem et au Van Leer Institute de Jérusalem,
I'historien Yariv Lapid a été de 2007 à 2013 directeur du service pédagogique du mémorial de Mauthausen. Il est aujourd'hui directeur du Centre humanisme et démocratie du musée des combattants des ghettos en Israël, où il propose des programmes pour un dialogue commun entre Arabes et Juifs.

\footnotetext{
(1) À propos du lieu de mémoire du camp de concentration de Mauthausen, voir l'étude sur les visiteurs de Das sozialwissenschaftliche Forschungsbüro, qui n'a été que partiellement publiée : Andreas Baumgartner et Isabella Girstmair, ,... weil ich das alles einmal sehen wollte." Die Mauthausen BesucherInnenStudie im Zuge der Neugestaltung der KZ-Gedenkstätte, in Bundesministerium für Inneres (Ed.), KZ-Gedenkstätte Mauthausen, Mauthausen Memorial 2010. Forschung, Dokumentation, Information, Vienne, 2011, p. 43-53.
}

\section{LA DYNAMIQUE DES VISITES DES LIEUX DE MÉMOIRE}

a visite d'un lieu de mémoire historique est tout particulièrement appréciée en complément des programmes d'enseignement actuels. Dans certains pays, le nombre de jeunes qui visitent des sites historiques est très élevé et peut même représenter la majorité des élèves. Ces visites ont une grande importance dans la socialisation et la construction identitaire, et jouent un rôle central dans les systèmes d'enseignement tant officiels qu'officieux.

Qu’en est-il cependant de la réalité concrète des visites de lieux de mémoire ? Comparées à l'ampleur du phénomène, les données permettant de sonder cette question sont relativement sommaires ${ }^{1}$ (Gudehus, 2006; Pampel, 2007). Une étude a toutefois été réalisée par l'Agence des droits fondamentaux de l'Union européenne (FRA), et publiée en $2010^{2}$.

J'aimerais mettre en exergue un constat important, à l'impact décisif sur la qualité d'une visite d'un lieu de mémoire : sa durée. L'étude de la FRA montre que plus de $90 \%$ des visiteurs y consacrent en moyenne deux heures. Cette indication est significative, car du seul fait de la taille de la plupart des lieux concernés, cette limitation dans le temps ne permet d'entreprendre que le parcours standard. D'autres activités telles que des séminaires nécessitent un temps supplémentaire que ne prend qu'un nombre très restreint de visiteurs - selon la conclusion à laquelle nous amène l'étude de la FRA.

Cela signifie que la principale activité que ces lieux de mémoire ont à offrir est le parcours standard sur leur site. Cela impliquerait que des moyens financiers adéquats soient employés à la configuration de ce parcours. Ce n'est malheureusement pas le cas. Les sommes investies dans les lieux de mémoire sont essentiellement destinées - outre l'entretien du site - à des expositions, des monuments mémoriels et des bâtiments d'exposition historiques. Les investissements dans des activités pédagogiques sont consacrés aux séminaires et aux programmes qui vont au-delà du parcours standard et nécessitent que les visiteurs restent plus de deux heures. Des 
sa topographie (vestiges physiques), et sa situation géographique, tout en tenan compte des différents profils des visiteurs (les groupes qui visitent le site). Pour atteindre cet objectif, l'équipe pédagogique a développé une méthode interactive en constante amélioration. Du fait de la taille et de l'histoire complexe du lieu, le temps de visite moyen ne concède que dix minutes environ à chaque station du parcours. Cela exige une méthodologie extraordinairement bien structurée - moins elle est structurée, plus le risque est grand qu’un des trois éléments disparaisse. Le transmetteur développe son récit grâce à ses contributions et à une structure. Chact lestich des lieux visités sur le site a une histoire spécifique. Le défi pédagogique consiste à creeer un cadre favorable à une discussion entre visiteurs concernant son histoire et sa signification. En utilisant délibe cadre pour un échange basé sur:

un contexte narratif minimal

- des documents

- des observations ciblées du site mémoriel

- des questions.

\section{LE CONTEXTE NARRATIF}

Le parcours guidé à travers le mémorial du camp de concentration de Mauthausen confronte les visiteurs à la question suivante : «Comment a-t-il été possible d'assassiner cent mille individus au cœur de la société dans un environnement civil? » Cette question est aujourd'hui le point d'ancrage de la visite du mémorial. Le récit du parcours repose explicitement sur l'hypothèse selon laquelle acteurs bourreaux et victimes étaient issus des classes moyennes de la société et que les camps de concentration n’auraient pas existé si la société n’y avait pas trouvé un intérêt, et n’y avait pas apporté son soutien actif. Le parcours accompagné est ainsi réparti en trois thèmes coïncidant avec la topographie du lieu de mémoire : il commence par l'environnement du camp (montrant qu'ilestintégré dans la société), s'intéresse au personnel du camp (les SS), et se termine par les victimes.

\section{a L’intégration du camp de concentration dans la société}

La première partie du parcours mène les visiteurs le long des murs du camp de concentration, jusquaa l'ancienne zone SS. Celle-ci se situe à l'extérieur des murs du camp et présente aux visiteurs des faits historiques qui contredisent ceux généralement admis. Ainsi, des éléments majeurs du camp de concentration, par exemple le camp sanitaire et la carrière se trouvaient à l'extérieur de ses murs, et rien ne les cachait à la vue de la population locale qui vivait à proximité. Des milliers de personnes ont été assassinées là où l'on voit aujourd'hui de paisibles prairies. La carrière, lieu de torture et d'assassinat de milliers de personnes, a, des la e dès la fin de la considération largement répandue dans l'opinion publique autrichienne est que les atrocités se déroulaient derrière les murs, invisibles et inaccessibles à la raison. Mais la réalité était toute autre : le camp a été érigé au cœur de la population civile, et conçu pour en être partie intégrante. Les maisons de la ville de Mauthausen n'en étaient éloignées que de quelques centaines de mètres. En excluant ces parties du camp de concentration, l'aménagement du lieu de mémoire conforte ce refoulement populaire répandu, et pérennise le discours «Nous n’avons rien vu et nous ne savions rien » d'après-guerre.

Durant plusieurs décennies, la visite du lieu commençait au niveau du portail d'entrée du camp. On occultait ainsi le camp sanitaire et la carrière, et par là même la visibilité des meurtres de masse perpétrés au cœur du village, sous les fenêtres des maisons voisines. Le nouveau concept pédagogique a changé la donne : aujourd'hui, la moitié du parcours de deux heures se déroule à l'extérieur des murs du camp et illustre la très étroite intégration du camp de concentration dans la société.

\section{b Les bourreaux}

La deuxième partie du parcours est consacrée à l'ancien secteur SS, qui se situe aussi à l'extérieur des murs du camp. La plupart des bâtiments ont été détruits après la guerre. Actuellement, leur emplacement est occupé par des mémoriaux nationaux. Un bâtiment, celui de la SS-Kommandantur n’a pas été démoli, il abrite nationaux. Un bâtiment, celui de la SS-Kommandantur n’a pas été démoli, il abrite
aujourd'hui l'administration du site. Avec la destruction du secteur SS ont disparu aujourd'hui l'administration du site. Avec la destruction du secteur SS ont disparu des preuves tangibles et décisives de la réalité du camp de concentration, à l'instar du Sonderstandesamt (l'administration d'État civil), d’une écurie et du casino SS.
L'ouverture du site de mémoire après la guerre a généré des critiques locales ; des L'ouverture du site de mémoire après la guerre a généré des critiques locales ; des leur place sur le sol autrichien (Perz, 2006) $)^{5}$.Le discours officiel autrichien affirmait que le national-socialisme était un phénomène allemand dont l'Autriche avait été la victime: elle ne pouva la victe nommis par le nationa autrichienne nétait pas moins inpliquée dans l'État nazi que la société allemande et qu'elle n’avait pas moins soutenu sa politique.

Des bâtiments comme celui du Sonderstandesamt illustrent en partie cette intégration. Lors d'un entretien filmé ${ }^{6}$ avec trois femmes âgées de la ville de Mauthausen, l'une d'entre elles décrit les yeux brillant son mariage avec un SS. Elle plonge dans les souvenirs de son charmant mariage et du bel orchestre; le tout se déroulant dans le secteur SS, à 30 mètres du portail d'entrée du camp de concentration. Elleévoque les nombreux et agréables SS à la fière allure, et révèle ainsi la normalité des relations desonépoque, arfón Les officies l'école du village, ils participaient aux manifestations culturelles locales. La SS avait

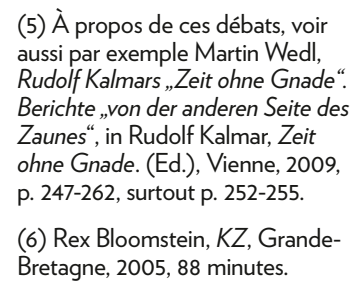


e équipe de football engagée dans la ligue locale, et on venait les voir dispute des matchs sur le terrain de sport qui faisait face au camp sanitaire. Tout cela allai parfaitement de soi : on était dans un village au cœur du Reich allemand, et non dans un environnement étranger.

L’image courante des SS - telle que la dessinent aujourd'hui encore les élèves qui visitent les lieux de mémoire - est celle d'hommes craints de tous. Elle est un pilier du mythe autrichien de la victime, qui décrit les SS comme des êtres brutaux et terrifiants qu'aucun être sain d'esprit ne se serait risqué à contredire. On ne repréd’intégrer ni les SS sous les traits d'adorables grands-pères.

\section{c Les victimes}

Pour la troisième partie du parcours, le visiteur franchit le portail d'entrée du camp de concentration et entre dans lancien camp de détention. C'est cette zone que les visiteurs perçoivent aujourd'hui comme le camp de concentration; elle comprend les baraques des détenus, une série de bâtiments de fonction telles que la buanderie et la cuisine, et une lace d'exécution. Différentes méthodes d'exécution ontétéutilietlaciine sées au camp de concentration de Mauthausen - dont le gazage, raison pour laquelle une chambre à gaz se situe dans cette partie. Lors de l'aménagement de ce lieu de mémoire, la plupart des baraquements existaient encore. Tous ont été détruits, à l'exception de trois bâtiments longeant la place d'appel. Avec les quatre bâtiments de l'autre côté de la place (la buanderie avec à la cave, les douches des détenus, la cuisine, la prison du camp et l'infirmerie avec le crématoire, et les installations d'exécution dans une cave commune), ils constituaient la représentation que l'on se faisait du camp de concentration de Mauthausen. En conséquence, les parcours se concentraient par le passé sur les victimes et visaient à susciter une identification à leur souffrance. A ces fins, on fournissait des descriptions manifestes de brutalité qu devaient choquer les visiteurs; par exemple, en décrivant les réactions phy au zyklon B à des jeunes de 14 ans, dans la chambre à gaz

L'identification aux victimes, l'empathie et la solidarité envers la souffrance humaine sont moralement sensées et nécessaires. Au mémorial concentrationnaire de Mauthausen, on avait - et on a encore - tendance à susciter chez le visiteur la fausse impression qu'il pouvait se représenter l'horreur du camp de concentration - ce qui entraîne des simplifications superficielles : les visiteurs sont ainsi généralement automatiquement du côté des victimes, ce qui fait émerger une communauté imaginaire de victimes. Dans un contexte de refoulement général de la responsabilité des atrocités ou de la collaboration - non seulement en Autriche, mais de manière générale en Europe -, la création de telles chimères est problématique. Dans le contexte spécifiquement autrichien, cela met en avant le mythe de l'Autriche «nation victime».
Dans l'élaboration de notre travail pédagogique, cette partie du parcours nous a valu de grandes difficultés. Et pourtant, les parcours actuels s'efforcent d'éviter les problèmes décrits, en essayant d'être précis sur le lieu, le moment d'utiliser des descriptions sobres, et la façon d'identifier les faits. Nous en donnons quelques exemples ci-après. L’introduction donnée par les transmetteurs à chaque station doit créer un contexte permettant aux visiteurs de comprendre les circonstances historiques des divers endroits visités. Le défi consiste à fournir uniquement des informations pertinentes clairement structurées etbien formulées, età éviter de longs discours. pertis cheours. On oublie aisénent que les visiteurs ne viennent pas pour nous écouter, mais pour visiter le lien de ménoire. Nous devons donc les aider à décrypter ce quils tout en évitant de devenir le centre delattention. Un défi particulier étant donné que bien des visiteurs - voire la plupart - viennent mal préparés. On cède facilement au besoin d'élargir leur horizon, de livrer de plus amples informations contextuelles et l'on se retrouve alors rapidement au milieu d'une histoire infiniment captivante de quinze minutes, et toute l'attention tournée vers nous.

Mais au final, nous n’avons rien dit de ce lieu très spécifique où nous nous trouvons, et sommes contraints de laisser de côté plusieurs parcours afin que les groupes regagnent leur car à temps. Les visiteurs n’arrivent pas totalement ignorants. Ils ont entendu parler du national-socialisme et des SS, et savent que dans les camps de entences camps de concentration ces $\mathrm{SS}$ ont infligé des sévices effroyablesà dautres individus - en général, les visiteur's de raconter Ihistoire par le menu dansle cadre d'un parcours de deux heures, la mise à disposition d'informations brèves et percutantes joue un grand rôle.

\section{DOCUMENTS (TEXTES, PHOTOS, ETC.)}

Après la contextualisation, l'étape suivante est l'observation de l'environnement de la section spécifique du parcours ou la présentation de documents. La documentation historique joue un rôle majeur dans la narration de l'histoire. Pour aider les visiteurs à comprendre la réalité historique, on utilise tout au lon du parcours des documents - textes ou photos. Les textes sont des descriptions du parcours des docunents - textes ou photos. Les textes sont des descriptions personnelles d'un incident ou d'une situation vécus par leur auteur à l'intérieur ou à l'extérieur du camp de concentration. Cet auteur ouvre ainsi une fenêtre étroite sur le passé ; un instantané qui nous permet de regarder le site du mémorial avec d'autres yeux. Les clichés aériens pris en mars 1945 par les Alliés jouent un rôle similaire. L'utilisation de documents peut répondre à différents objectifs si les textes sont choisis avec soin (ce qui ne peut être présupposé). On est ainsi plus précis sur le plan historique et l'on s'appuie sur l'autorité d'une source historique. On y gagne en outre en concision, ce qui est difficile à obtenir par le biais d'un discours libre Car dans le format de visite moyen du site de mémoire, un parcours compé d'environ deux heures impliquant de couvrir un domaine ties vaste accors the et d'aborder des thènes extrênenent complexes, la concision est d une importance 

documents a une ambition plus large.

Un important défi méthodologique consiste à trouver des textes renferman quelques-uns des aspects moraux auxquels est confronté le parcours du mémorial. Citons en exemple le texte suivant, propre à illustrer la routine sociale et le fait que violence, torture et meurtre étaient connus : Eleonore Gusenbauer, agricultrice de Marbach (commune de Ried dans le Riedmark) :

Au camp de concentration de Mauthausen, des détenus ont de nouveau été fusillés au niveau de la carrière Wiener Graben ; ceux qui ne sont pas morts sur le coup vivent encore un moment, allongés à côté des morts, des heures voire des demi-journée entières. Ma propriété se situe sur une butte à proximité de cette carrière, et l'on est souvent témoin involontaire de ces exactions. Je suis souffrante et cette vue met mes nerfs tellement à lépreuve que je n’arriverai pas à endurer cela très longtemps. Je demande que de tels actes inhumains soient réprimandés, ou perpétrés là où on ne les voit pas.

La plainte de Madame Gusenbauer témoigne del'intégration du camp de concentration dans la société du Troisième Reich. Elle permet à la fois de comprendre la tration dans la société du Troisième Reich. Elle permet à la fois de comprendre la réalité historique et de remettre en question les fréquentes suppositions quant à la collaboration de la société. Une fois ces documents montrés, létape suivante serait une discussion au sein du groupe sur la signification de ces faits historiques pour nous présents aujourd'hui en ce lieu. Un atelier d'environ dix minutes est prévu à l'une ou l'autre station du parcours, par exemple au niveau de la carrière. Un tel atelier s’appuie sur les éléments structurels suivants : l'utilisation de documents, la narration d'un contexte, l'observation du mémorial et un moment pour la prise de questions. Afin d'optimiser la participation des visiteurs, la présentation des documents doit être bien réfléchie et bien préparée : format du papier (A4 ou A5), couverture plastifiée et distribution aux participants sont autant de facteurs-clés (A) en vue dunediscas documents, en particulier quand ls soulèvent de nombreuses questions. Lobservation de photos exige plus qu'un simple regard furtif si l'on veut veritablement s'en imprégner. La distribution du nombre exact d'exemplaires aux participants pou leur permettre de travailler isolément, mais aussi de discuter en petits groupes (un exemplaire pour trois) joue un rôle important dans leur interprétation. Le document historique en mains, les participants sont moins dépendants d'un transmetteur, source de connaissances et de compréhension. La documentation historique peut et doit être interprétée. L'accès immédiat à un document historique permet aux visiteurs de mieux réféchir de facon autonome et d'en juger l'interprétation et l'importance. Avoir à disposition le document aide à s'y confronter et à l'interpréter - et ainsi à interpreter l'histoire (il confirme donc aussi la responsabilité
des participants).

\section{OBSERVATIONS}

Quand ils se tiennent sur l'ancien terrain du camp de concentration, avec vue, vers le bas, sur la carrière Wiener Graben - où des détenus étaient assassinés tous les jours - et, vers le haut, sur la maison d'Eleonore Gusenbauer, conformément à la description du document, les visiteurs peuvent avoir un aperçu d'une réalité historique du camp de concentration et de son environnement. Associer documentation et observation du terrain, deux pratiques liées par le contexte, perme aux visiteurs de se faire une idee de la situation historique. Plonge dans son travail au méno dal concentrationnaire que les visiteurs y viennent pour la première fois et, généralement, la dernière À force de répéter ce parcours, il a tendance à oublier ce que l'on ressent quand on ne l'a encore jamais traversé. Il néglige ainsi souvent de donner aux visiteurs suffisamment de temps pour observer ce qui les entoure, et de les aider à voir ce qui est invisible au premier regard et qui lui saute aux yeux. Il en va de même du traitement des documents ; les visiteurs ont également besoin de temps pour comprendre un document, voir les détails d'une photo ou lire une phrase à deux ou trois reprises pour bien la comprendre.

De façon similaire à l'utilisation de documents, l'observation détourne l'attention du transmetteur « seule source d'information ». Elle permet aux visiteurs d'accéder immédiatement aux thèmes en présence et augmente autant le nombre que la nature des documents dont les visiteurs tirent leurs informations. On utilise ainsi quelques-uns des fondements de la formation interdisciplinaire, qui amènent à communiquer des informations par le biais de canaux autres que la seule voix des transmetteurs. L'observation du site et de son environnement, la lecture de textes de genres et styles différents, l'observation de photos-portraits ou de la topographie augmentent les possibilités de transmission d'informations.

Le terrain de sport et le camp sanitaire du camp de concentration - situés côte à côte - illustrent l'importance de l'observation. Bien que le mémorial ait été ouvert aux visiteurs en 1949, ce secteur a été intégré au parcours il y a quelques années seulement. Des millions de visiteurs n'ont guère eu la possibilité d'y accéder, et leur attention n’a pas été attirée sur ce lieu où des milliers de détenus ont trouvé la mort tandis que d'autres venaient y jouer au football ou regarder des matchs. Aujourd'hui, le terrain de sport et le camp sanitaire sont une seule et magnifique prairie. Si notre regard n’y était pas attiré, si l'on ne nous donnait pas à voir des photos montrant le terrain de sport et les baraques du camp sanitaire, on en viendrait à ignorer ces éléments de la réalité du camp de concentration. Les visiteurs qui en cours de visite guidée se tiennent près de la prairie et regardent des clichés aériens peuvent sentir a proximité entre le divertissementetla mort. lls peuventconstaterqueles gensqui laproxich venaient y rearder un match de football ne pouvaient faire autrement que de voir 
Pédagogie des lieux de

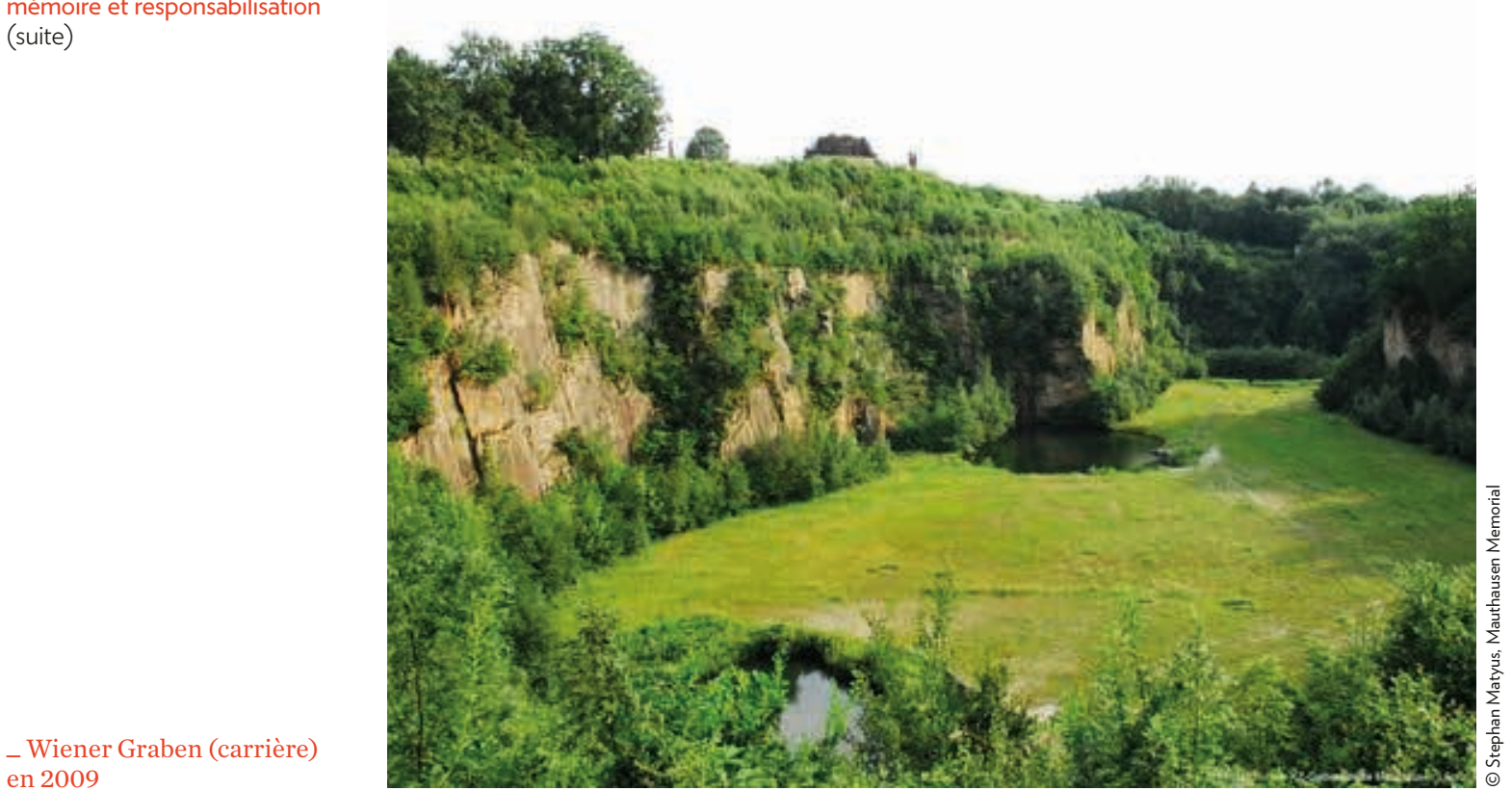

en 2009

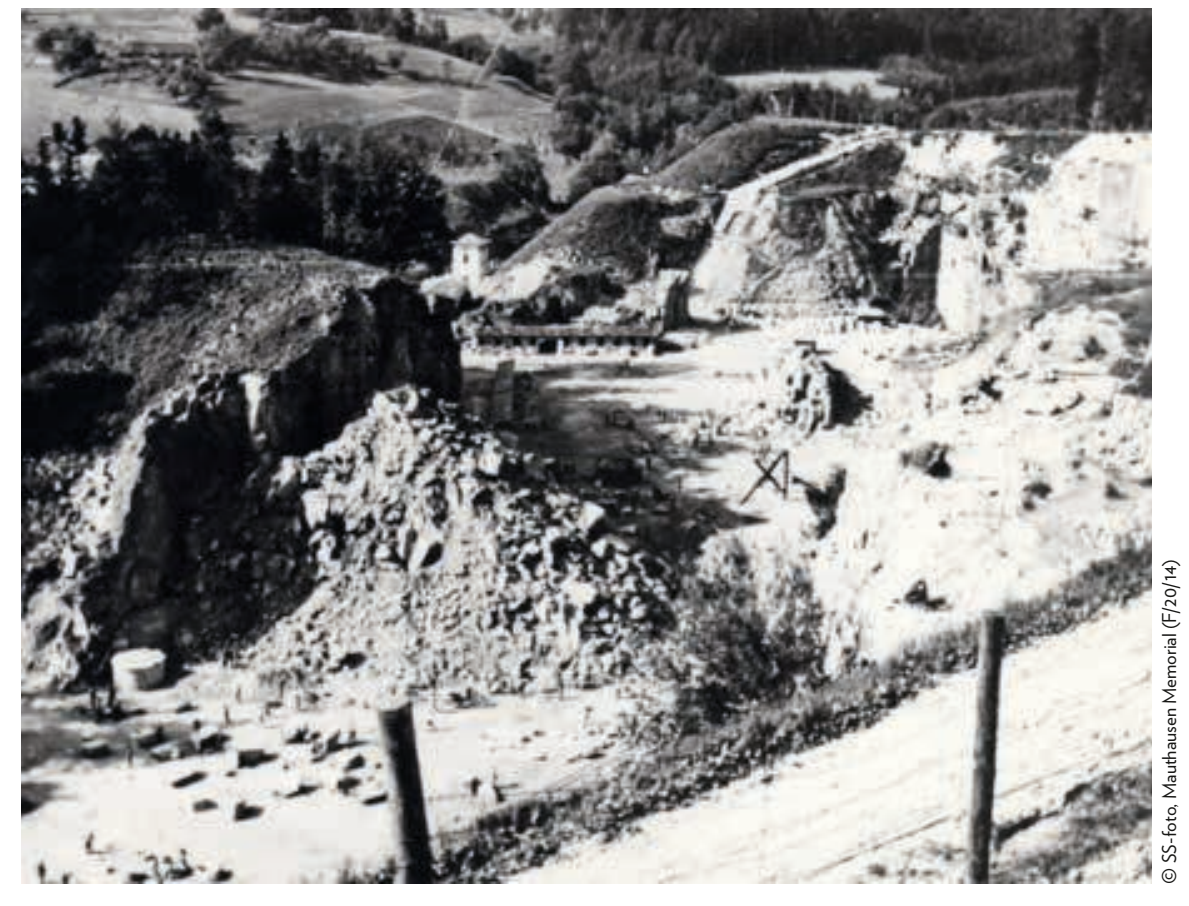

de 1944 décrivant un match d'une ligue locale de football au camp de concentration confère à l'image historique une plus grande profondeur.

\section{QUESTIONS}

L’image émergeant des documents intégrés au parcours est inquiétante. Elle est difficile à accepter - en particulier pour les individus issus de la société dont provient cette imà Au-delà de la diffeulté qu'il y a d'imaginer sa propre famille pre famille pouvoir y être rest également opposé à l'environnement que permet de découvrir la visite. D’après ce récit, les atrocités se déroulèrent derrière des murs, loin des yeux du public, et étaient perpétrées par des hommes qui n'avaient pas de lien de parenté avec nous. En confrontant de plus près les visiteurs avec ces informations déconcertantes - et ainsi en remettant en question et en sapant les récits et hypothèses dominants -, les transmetteurs peuvent évaluer avec les visiteurs leurs implications et leur signification. Pour y parvenir, il doit les inviter à réfléchir en posant des questions. Notre expérience montre que formuler une question pertinente est une tâche très difficile. Ainsi, les questions relatives au sens età l'interprétation sont difficiles à énoncer. Nous sommes enclins à poser des questions sur des faits, des questions fermées dont ils connaissent déjà la réponse estions suggestives qui guident lesp préétablies. Comme je l'ai déjà évoqué au début, ils sont encore souvent impuissants quant aux implications de ces résultats de recherche, en dépit des nombreuses infor-

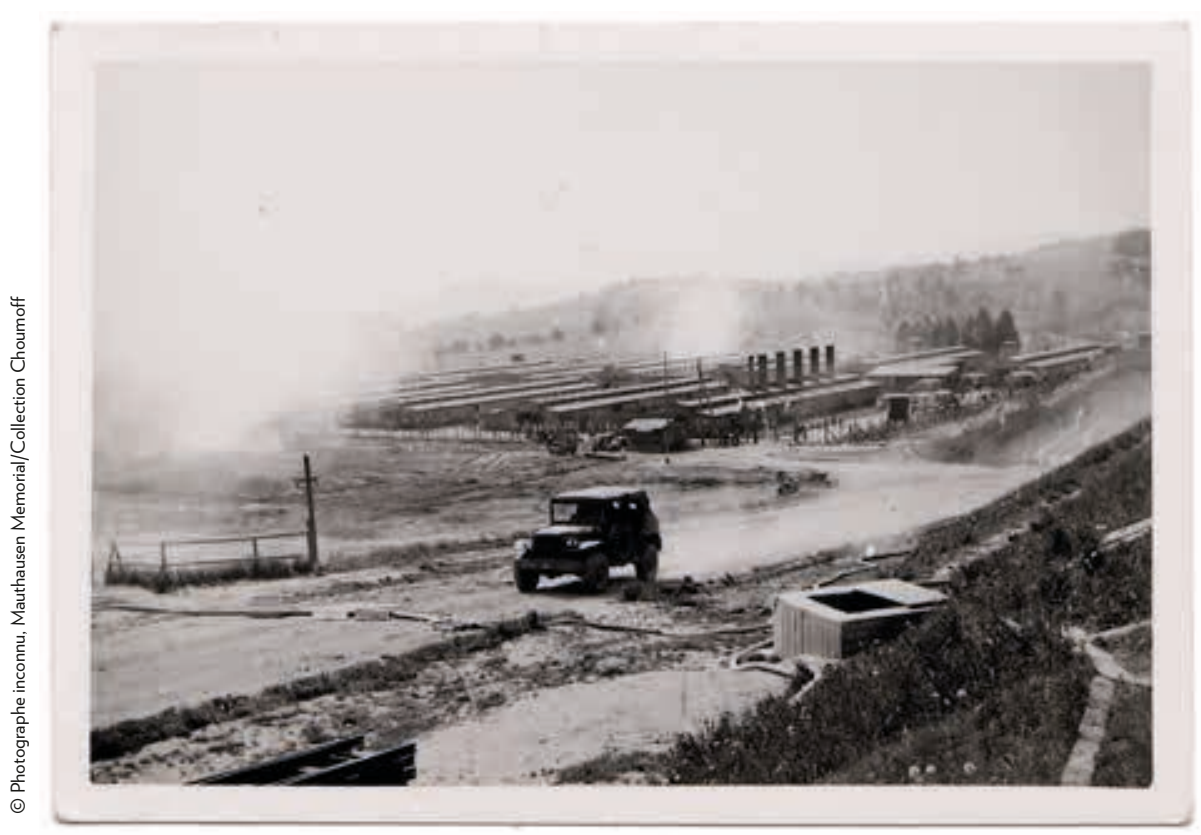

- Terrain de sport SS (en haut à gauche) et le « camp plan), tout de suite après la plan), tout 
Pédagogie des lieux de

mémoi
(suite)

mations historiques fournies par les chercheurs. La scène du match de football en face du camp sanitaire nous permet d'ouvrir sous certains angles la discussion dans le débat public sur les sites de mémoire.

Cherchant à définir ce qu'est une bonne question, nous avons élaboré quelques hypothèses. Une bonne question serait ainsi pour nous :

- une question qui a plus d’une réponse possible,

- une question qui stimule l'interprétation et la réflexion critique,

- une question qui préoccupe réellement celui qui la pose, et qu'il ne pose pas uniquement pour dire quelque chose.

L'écart entre les faits (les informations données aux visiteurs) et la question ('introduction à une discussion) peut être comblé par des questions qui éveillent l'imagination. Cela s'avère souvent nécessaire compte tenu du défi émotionnel et intellectuel qu'un tel cadre provoque. On pourrait citer par exemple une question relative aux matchs de football près du camp de concentration de Mauthausen : «Imaginons ce que voyaient les gens qui venaient assister autrefois à un match quand ils regardaient au-delà du terrain de football et voyaientles détenus exsangues derrière les barbelés du camp sanitaire. »

Le processus de représentation suscite une réflexion historique, un jugement moral et une interprétation. Cette question ayant plusieurs réponses possibles, elle entraînera idéalement une discussion entre les visiteurs. Une autre interrogation serait: Comment les hommes sont-ils devenus aveugles à la souffrance d'autrui, au point de pouvoir s'amuser en même temps qu'ils en étaient témoins ?

C'est par cette question, qui englobe une plus ample connaissance des atrocités dans la société et l'analyse psychosociale de leurs circonstances, que je clôturerai ce texte sur la mise au point du concept pédagogique du mémorial concentrationnaire de Mauthausen. I

Traduit de 'allemand par Emilie Syssau

BIBLIOGRAPHIE

Christian Gudehus, Dem Gedächtnis zuhüren. Gedenkstätten und
Erzählungen über nationalsozialistische Verbrechen, Essen, Klartext. 2006.

- Yariv Lapid, Christian Angerer, Maria Ecker, «, Was hat es mit mir zu tun?" Das Vermittlungskonzept an der Gedenkstätte Mauthaus
Gedenkstätten Rundbrief no 162,8 novembre 2011, p. 40-45.
- Bert Pampel, ,Mit eigenen Augen sehen, wozu der Mensch fähigi ist": Zur
Wirkung von Gedenkstätten auf ihre Besucher, Francfort/Main, Campus, 2007. - Bertrand Perz, Die KZ-Gedenkstätte Mauthausen 1945 bis zur Gegenwart,
Innsbruck, Studien, 2006.

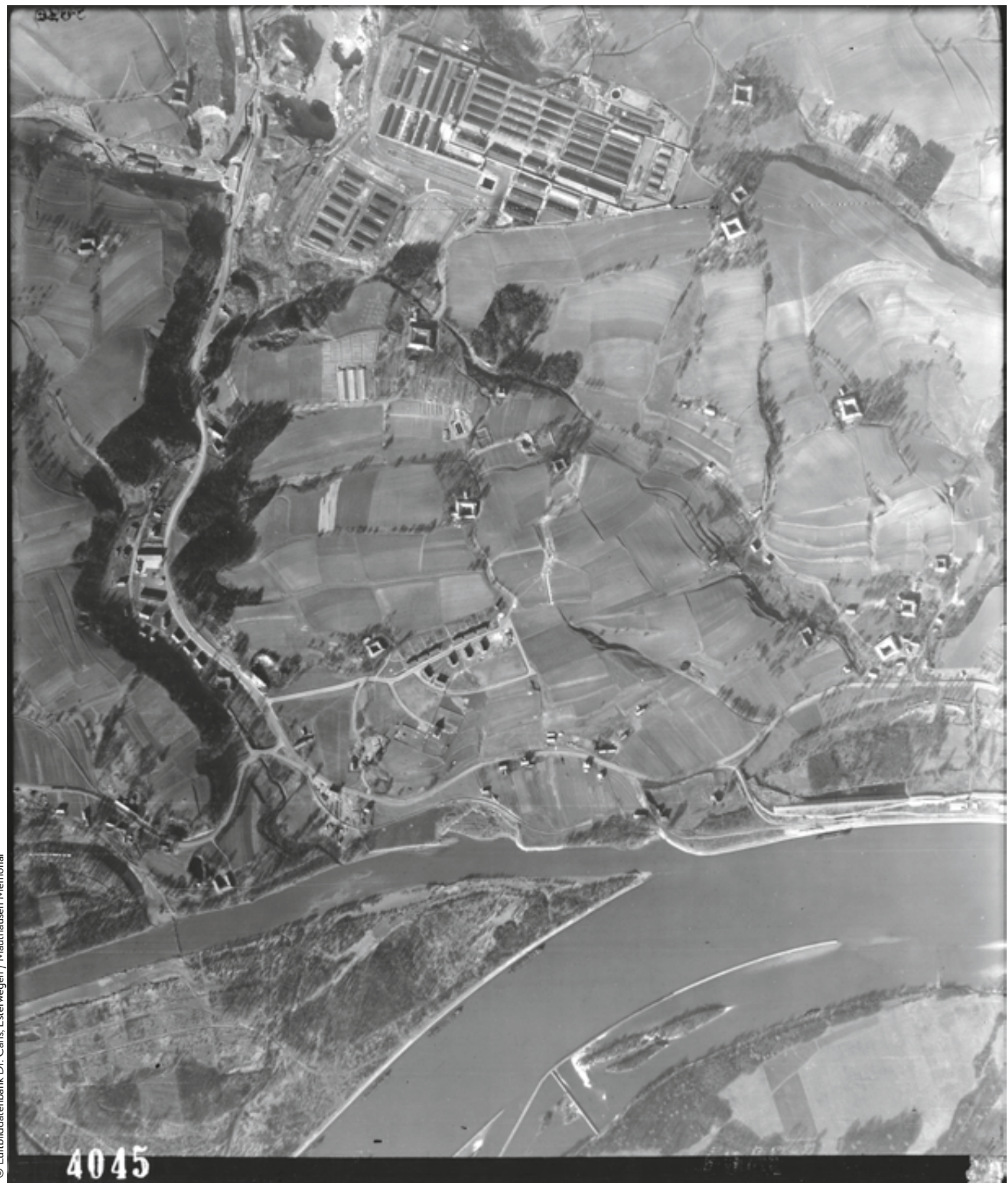

